\title{
Evidence of Lipid Degradation During Overnight Contact Lens Wear: Gas Chromatography Mass Spectrometry as the Diagnostic Tool
}

\author{
Amandeep Panaser and Brian J. Tighe \\ Aston University, Biomaterials Research Unit, Bio-Materials and Molecular Science Group, Aston, Birmingham, United Kingdom
}

Correspondence: Amandeep Panaser, Aston University, Biomaterials Research Unit, Bio-Materials and Molecular Science Group Aston, Aston Triangle, Birmingham B4 7ET, $\mathrm{UK}$;

panasera@aston.ac.uk.

Submitted: July 22, 2013

Accepted: February 13, 2014

Citation: Panaser A, Tighe BJ. Evidence of lipid degradation during overnight contact lens wear: gas chromatography mass spectrometry as the diagnostic tool. Invest Ophthalmol Vis Sci. 2014;55:1797-1804. DOI:10.1167/ iovs.13-12881
Purpose. We investigated structural differences in the fatty acid profiles of lipids extracted from ex vivo contact lenses by using gas chromatography mass spectrometry (GCMS). Two lens materials (balafilcon A or lotrafilcon A) were worn on a daily or continuous wear schedule for 30 and 7 days.

MeтноDs. Lipids from subject-worn lenses were extracted using 1:1 chloroform: methanol and transmethylated using 5\% sulfuric acid in methanol. Fatty acid methyl esters (FAMEs) were collected using hexane and water, and analyzed by GCMS (Varian 3800 GC, Saturn 2000 MS).

Results. The gas chromatograms of lens extracts that were worn on a continuous wear schedule showed two predominant peaks, C16:0 and C18:0, both of which are saturated fatty acids. This was the case for balafilcon $\mathrm{A}$ and lotrafilcon $\mathrm{A}$ lenses. However, the gas chromatograms of lens extracts that were worn on a daily wear schedule showed saturated (C16:0, C18:0) and unsaturated (C16:1 and C18:1) fatty acids.

Conclusions. Unsaturated fatty acids are degraded during sleep in contact lenses. Degradation occurred independently of lens material or subject-to-subject variability in lipid deposition. The consequences of lipid degradation are the production of oxidative products, which may be linked to contact lens discomfort.

Keywords: lipid degradation, contact lens discomfort, overnight contact lens wear
M any analytical techniques have been used for the analysis of lipids in the ocular environment. A recent publication by Pucker and Nichols ${ }^{1}$ reviews the array of techniques used for meibomian and tear lipid analysis. While gas chromatography (GC) and gas chromatography mass spectrometry (GCMS) have been used for meibomian lipid analysis, they have not been used to analyze changes in the fatty acid component of lipids resulting from contact lens wear. Iwata et al. ${ }^{2}$ used it to evaluate lipids adsorbed on silicone hydrogel lenses in vitro. The GCMS is a powerful technique used for the detection of individual species at low concentrations.

The lipid composition of meibomian secretions and tears has been investigated by many researchers ${ }^{3-19}$; however, less attention has been paid to the fatty acid component of tear lipids. ${ }^{18,20-23}$ Fatty acids are a key building-block of lipids; it is their length and degree of unsaturation that dictate oxidative stability. Tear lipids, in their native environment are oxidatively stable because meibomian glands predominantly secrete only saturated and monounsaturated lipids. This contrasts with other interfaces in the body where polyunsaturated acids are prevalent. ${ }^{24}$ Nicolaides et al. ${ }^{18}$ examined the meibomian fatty acid composition and found that the most predominant fatty acids secreted are C16:0, C18:0 (saturated, no double bonds), C16:1, and C18:1 (monounsaturated, one double bond). The tear sampling method used in this case was expression of the meibomian glands together with gas liquid chromatography (GLC) to analyze samples. Joffre et al. ${ }^{21}$ reported that the fatty acid composition of meibum collected from healthy subjects was predominantly C16:0, C18:0, and C18:1, which agreed with the observations of Nicolaides et al. ${ }^{18}$

Recent publications by Butovich ${ }^{11,12}$ report the presence of very long chain fatty acids (VLCFAs, C30:0 and above) in human meibomian gland lipids, particularly in the form of cholesteryl esters. Long chain fatty acids (C16-C26) have been identified previously by several investigators ${ }^{21,23}$; however, the parent lipid classes have not been identified due the limitations of the analytical technique used. Butovich et al. ${ }^{14}$ also suggested that VLCFAs may have escaped identification by techniques, such as GCMS, because the high sample preparation and operating temperatures could lead to degradation of lipids. However, fatty acids in the form of fatty acid methyl esters (FAMEs) have been shown to be thermally stable at temperatures up to $325^{\circ} \mathrm{C}$, which provides the basis of a convenient method for fatty acid investigation. ${ }^{25}$ The GCMS sample preparation temperatures and column oven temperatures rarely exceed these temperatures, which suggest the suitability of this technique for the study of FAMEs.

The tear film composition of the open eye is very different from its composition during overnight eye closure. The overnight tear film becomes stagnant as a result of reduced tear flow and no tear exchange, resulting in compositional changes and nocturnal inflammatory characteristics. These characteristics, including the levels of tear proteins following overnight eye closure, with and without contact lens wear, have been investigated previously. ${ }^{26-28}$ In these studies microcapillary tubes were used for tear collection immediately after opening the eye and changes in the levels of tear proteins were 
measured. Microcapillary tear collection is a suitable method for aqueous tear collection; however, it is not ideal for tear lipid collection. The research in this study exploited the use of contact lenses as a probe, which allows significant levels of lipid to be collected. ${ }^{24}$ The contact lens can, thus, provide a "snapshot" of compositional changes occurring during sleep.

Contact lens wear can change tear film dynamics and, thus, influence tear lipid composition during wear. Individual tear chemistry, lens material, and wear schedule all influence lipid deposition. ${ }^{24}$ The factors affecting lipid deposition processes and extractability before analysis have been well studied. ${ }^{29-32}$ In this study, GCMS is used as the analytical tool to show how fatty acid composition of lipids is affected by these factors.

\section{Materials ANd Methods}

High performance lipid chromatography (HPLC)-grade solvents were used for the extraction of lenses and all subsequent sample preparation. The solvents were purchased from Fisher Scientific (Loughborough, UK).

\section{Tear Sampling and Extraction Methods}

Ophthalmic Sponge Tear Collection and Lipid Extraction. Tear lipids were collected by placing the tip of the ophthalmic sponge (BD Visispear eye sponge; Beaver-Visitec International, Oxfordshire, UK) in the lower meniscus of the eye, taking care to minimize contact with the conjunctiva. Tears were collected by the sponge, and the wetted tip of the sponge was cut off and placed in a glass vial for extraction. Then, $1.5 \mathrm{~mL}$ chloroform were added to the glass vial and the sponge was extracted for at least 2 hours on a flat-bed shaker. The sponge was removed, the extraction solvent evaporated to dryness, and the extract was transmethylated (described in the GCMS sample preparation section).

Microcapillary Tear Collection and Lipid Extraction. The microcapillary tube (Sigma-Aldrich, Suffolk, UK) was placed in the lower meniscus of the eye and tears were drawn into the tube by capillary action. The tears and tube were extracted in $(1.5 \mathrm{~mL}) 1: 1$ chloroform:methanol for at least 40 minutes. The extract solvent was evaporated to dryness and transmethylated (described in the GCMS sample preparation section).

Ex Vivo Contact Lens Extraction. Single lenses were extracted in $(1.5 \mathrm{~mL}) 1: 1$ chloroform:methanol for at least 40 minutes in a glass vial. The extract solvent was evaporated to dryness and transmethylated (described in the GCMS sample preparation section).

Various extraction times and solvents have been described in the literature. ${ }^{33,34}$ The conditions used here were optimized from a range of experimental conditions involving single and multiple extractions.

\section{GCMS Sample Preparation and Operating Conditions}

Preparation of FAMEs. The extract was transmethylated for 90 minutes at $70^{\circ} \mathrm{C}$ in a dry block by adding methanol and sulfuric acid (5\%). The FAMEs were extracted using hexane and water. The tubes were shaken by hand and allowed to stand for phase separation. The upper layer was collected and transferred to an amber vial, and the FAMEs were analyzed by GCMS.

GCMS Conditions. A Saturn Varian 2000 GCMS system with an autosampler was fitted with a VF-5MS column (Agilent Varian, Berkshire, UK). The column temperature program used was an initial temperature of $120^{\circ} \mathrm{C}$ for 2 minutes, increased to $200^{\circ} \mathrm{C}$ at $10^{\circ} \mathrm{C} / \mathrm{min}$, and held for 1 minute, and then increased to $250^{\circ} \mathrm{C}$ at $10^{\circ} \mathrm{C} / \mathrm{min}$ and held for 19 minutes. The sample was injected using a splitless method. The injection port temperature was set at $250^{\circ} \mathrm{C}$. Helium was used as the carrier gas and the flow rate was set at $1.0 \mathrm{~mL} / \mathrm{min}$. The mass spectrometer was set on the electron ionization (EI) mode and the ionization range was set between 40 and $650 \mathrm{~m} / \mathrm{z}$. The sample analysis time was 35 minutes. Peak areas of repeated injections were reproducible to within $\pm 5 \%$.

\section{Daily Wear Versus Continuous Wear: Contact Lens Samples and Clinical Study Details}

This study was conducted according to the guidelines laid down in the Declaration of Helsinki and all procedures involving human subjects were approved by the Aston University Human Sciences Committee.

The study involved 43 subjects wearing either lotrafilcon A or balafilcon A lenses for 30 days, on either a daily or continuous wear schedule (daily plus overnight wear). Each of the four subgroups contained 11 subjects $( \pm 3)$ with matched average ages of 20 years ( \pm 1 year). The overall male-to-female ratio was 26:17 and was balanced evenly in each subgroup.

The lenses worn on a daily wear basis were removed at the end of the day, cleaned, disinfected, and stored in Opti-Free Express (Alcon Laboratories, Inc., Ft. Worth, TX). For those subjects who showed sensitivity to Opti-Free Express, ReNu Multiplus (Bausch \& Lomb, Inc., San Antonio, TX) was used.

\section{Results and Discussion}

Initially, tear samples collected using microcapillary and ophthalmic sponges, and lipids extracted from ex vivo conventional hydrogel lenses were analyzed using GCMS. Analyzing tear samples and preliminary lens samples allowed GCMS to be validated as a suitable technique for the analysis of changes in fatty acid profiles with wear schedule.

Figure 1 shows the information that can be obtained from individual chromatograms. Individual peaks on chromatograms were identified by their characteristic retention time and mass spectra (Fig. 1, inset). Smaller chain fatty acids had shorter retention time and larger chain fatty acids had longer retention times ( $x$-axis). The retention time for the equivalent unsaturated fatty acid was slightly faster; therefore, C16:1 had a faster retention time compared to $\mathrm{C} 16: 0$ and $\mathrm{C} 18: 1$ also had a faster retention time compared to C18:0.

The concentration of individual fatty acids was determined from the peak area, which correlated directly with peak intensity (on the $y$-axis). Specific areas of the chromatogram could be zoomed-in on to view individual peaks in more detail. The mass spectra for individual peaks also could be obtained, which allowed the identity of the peak to be determined accurately. Molecular weight and structural information were provided from the mass spectra of individual peaks (Fig. 1, inset).

Fatty acid profiles varied depending on the sampling technique used for lipid collection (Table 1). Low levels of gross lipid were collected using the microcapillary method (peak heights very low); however, the sponge was a better choice for tear-lipid collection. The fatty acid profiles of ex vivo lens extracts and tear lipids collected by the ophthalmic sponge were similar. The predominant fatty acids observed for the majority of the lens extracts and tear samples were C14:0, C16:1, C16:0, C18:1, and C18:0 (Table 1).

Although the ophthalmic sponge appeared to be a good method of tear-lipid collection, in some cases it was difficult to obtain a tear sample using this technique because of low tear 


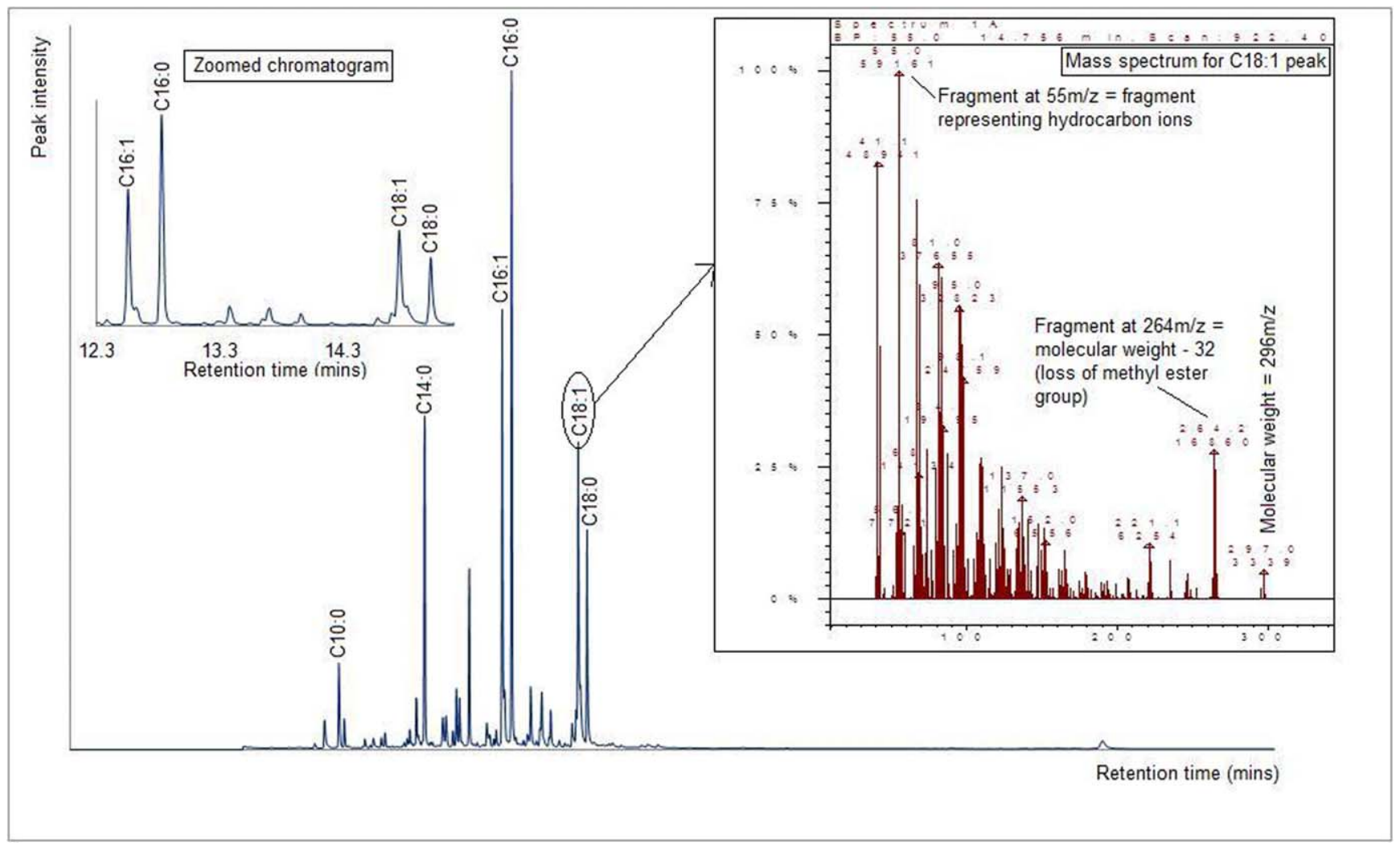

FIGURE 1. A "generic" GC trace of standard fatty acid methyl esters and the information obtained from an individual GC trace.

flow. Lens extracts were the lipid sampling method of choice for this research, since lipids accumulate on all lenses, and they can be extracted and analyzed readily.

\section{Ex Vivo Balafilcon A and Lotrafilcon A Lens Extracts: 30 Days Daily Wear Versus Continuous Wear}

Ex vivo balafilcon A and lotrafilcon A lenses, worn with two different wear schedules, each for 30 days, were extracted and analyzed by GCMS. The GC traces showed significantly different fatty acid profiles for lens extracts that were worn on a daily wear schedule compared to those worn on a continuous wear schedule. The difference was dramatic.

Lenses worn by several subjects were analyzed $(n=22)$ and the results summarized in Table 2. For greater clarity, representative chromatograms are displayed (Figs. 2, 3) which typify the fatty acid profiles that were observed for lens extracts of the range of subjects. Although lipid class profiles are known to differ from subject to subject, ${ }^{24}$ the profiles of the fatty acids that constitute the lipid ester classes were

LOD, limit of detection. similar for the range of subjects. One observed point of difference, however, was that the ratio of unsaturated to saturated fatty acids did show some variation. This is reflected in the subject-to-subject variations for lenses worn on a daily wear schedule (Table 2).

C18:0 and C18:1 were chosen as the saturated and unsaturated markers, because they are the predominant fatty acids secreted by meibomian glands according to previous literature $^{18}$ and observed to be the dominant peaks in the current study. A useful comparison of the ratio of unsaturated to saturated fatty acids can be obtained directly from the heights of C18:0 and C18:1 peaks from the GC traces of lens extracts for various subjects wearing the two lens types for two wear schedules. The results are summarized in Table 2 .

Table 2 shows that for the majority of subjects the proportion of saturated fatty acids found in daily wear lenses is rather greater than that of unsaturated fatty acids (an average of 1.7 to 1 from the aggregated data for all lenses). However, for a small minority of subjects (in both lens materials) a significantly higher ratio $(>2: 1)$ of unsaturated to saturated fatty acid was observed. In addition to subject-to-subject

TABLE 1. Comparison of Fatty Acid Detection Profiles Obtained From Tear Samples and Contact Lens Extracts

\begin{tabular}{cclccc}
\hline $\begin{array}{c}\text { Peak Retention } \\
\text { Time, min }\end{array}$ & $\begin{array}{c}\text { Peak } \\
\text { Identity }\end{array}$ & $\begin{array}{c}\text { Saturated/ } \\
\text { Unsaturated }\end{array}$ & $\begin{array}{c}\text { Microcapillary } \\
\text { Tear Sample }\end{array}$ & $\begin{array}{c}\text { Visispear Sponge } \\
\text { Tear Sample }\end{array}$ & $\begin{array}{c}\text { Ex Vivo Conventional } \\
\text { Hydrogel Lens Extract }\end{array}$ \\
\hline 10.3 & C14:0 & Saturated & $\times$ below LOD & $\checkmark$ & $\checkmark$ \\
12.6 & C16:1 & Unsaturated & $\times$ below LOD & $\checkmark$ & $\checkmark$ \\
12.8 & C16:0 & Saturated & $\checkmark$ (low levels) & $\checkmark$ & $\checkmark$ \\
14.8 & C18:1 & Unsaturated & $\checkmark$ (low levels) & $\checkmark$ & $\checkmark$ \\
15.0 & C18:0 & Saturated & $\checkmark$ (low levels) & $\checkmark$ & $\checkmark$
\end{tabular}


TABLE 2. Ratios of C18:1 (Unsaturated) to C18:0 (Saturated) Fatty Acids Determined From the GC Traces of Lens Extracts From the Range of Subjects Studied

\begin{tabular}{|c|c|c|c|c|c|}
\hline Subject & $\begin{array}{l}\text { Peak Height C16:1, } \\
\text { Counts } \times 10^{3}\end{array}$ & $\begin{array}{l}\text { Peak Height C16:0, } \\
\text { Counts } \times 10^{3}\end{array}$ & $\begin{array}{l}\text { Peak Height C18:1, } \\
\text { Counts } \times 10^{3}\end{array}$ & $\begin{array}{l}\text { Peak Height C18:0, } \\
\text { Counts } \times 10^{3}\end{array}$ & $\begin{array}{c}\text { Ratio of } \\
\text { Unsaturated to Saturated, } \\
\text { C18:1-to-C18:0 }\end{array}$ \\
\hline \multicolumn{6}{|c|}{ Daily wear balafilcon A lenses } \\
\hline 1 (Fig. 2) & 275 & 1100 & 300 & 500 & $1: 1.5$ \\
\hline 2 & 50 & 220 & 150 & 170 & $1: 1.3$ \\
\hline 3 & 90 & 600 & 600 & 600 & $1: 1$ \\
\hline 4 & 100 & 900 & 300 & 800 & $1: 2.5$ \\
\hline 5 & 100 & 300 & 450 & 150 & $1: 0.33$ \\
\hline 6 & 190 & 600 & 300 & 600 & $1: 2$ \\
\hline Average & 134 & 620 & 350 & 470 & $1: 1.3$ \\
\hline $\mathrm{SD}$ & 82 & 338 & 154 & 259 & - \\
\hline \multicolumn{6}{|c|}{ Daily wear lotrafilcon A lenses } \\
\hline 1 (Fig. 3) & 22 & 120 & 60 & 140 & $1: 2.3$ \\
\hline 2 & 25 & 120 & 70 & 150 & $1: 2$ \\
\hline 3 & 10 & 150 & 50 & 175 & $1: 3.5$ \\
\hline 4 & 15 & 40 & 20 & 40 & $1: 2$ \\
\hline 5 & 7 & 100 & 275 & 120 & $1: 0.44$ \\
\hline 6 & 5 & 300 & 275 & 400 & $1: 1.5$ \\
\hline Average & 14 & 138 & 125 & 170 & $1: 1.4$ \\
\hline $\mathrm{SD}$ & 8 & 87 & 117 & 121 & - \\
\hline \multicolumn{6}{|c|}{ Average ratio of 18:1-to-18:0 fatty acid from aggregated data for all daily wear lenses: $1: 1.7$} \\
\hline \multicolumn{6}{|c|}{ Continuous wear balafilcon A lenses } \\
\hline 1 (Fig. 2) & - & 300 & 40 & 310 & $1: 8$ \\
\hline 2 & - & 80 & $<15$ & 79 & $1: 5$ \\
\hline 3 & - & 80 & $<15$ & 100 & $1: 7$ \\
\hline 4 & - & 40 & $<<15$ & 30 & $1:>2$ \\
\hline 5 & - & 400 & $<15$ & 200 & $1: 13$ \\
\hline 6 & - & 200 & $<15$ & 175 & $1: 12$ \\
\hline Average & - & 183 & 20 & 149 & $1: 7.5$ \\
\hline $\mathrm{SD}$ & - & 143 & - & 100 & - \\
\hline \multicolumn{6}{|c|}{ Continuous wear lotrafilcon A lenses } \\
\hline 1 (Fig. 3) & - & 72 & $<10$ & 50 & $1: 5$ \\
\hline 2 & - & 78 & $<10$ & 75 & $1: 7.5$ \\
\hline 3 & - & 120 & $<10$ & 150 & $1: 15$ \\
\hline 4 & - & 100 & $<10$ & 120 & $1: 12$ \\
\hline Average & - & 92 & 10 & 98 & $1: 10$ \\
\hline SD & - & 21 & - & 44 & - \\
\hline
\end{tabular}

differences, possible noncompliance in wear and lens care regime might contribute to the observed variations.

Although there are observed subject-to-subject differences in the relative proportions of C18:0 and C18:1 fatty acids, these are small compared to the differences between extracts from daily and continuous wear lenses. The data in Table 2 clearly demonstrated this difference over a range of subjects in both lens materials. For daily wear lenses, the ratio of unsaturated to saturated lipids rarely exceeds 1:2; however, for continuous wear lenses the average ratio is approximately $1: 9$. This marked difference reflects a dramatic reduction in the concentration of extractable unsaturated C18:1 fatty acid found on continuous wear compared to daily wear lenses.

It is clear that there are complicating factors that cloud statistical analysis of the data set shown in Table 2 . The plasma oxidized surface of balafilcon A lenses is very different from the plasma-coated lotrafilcon A surface. In consequence, lipoidal deposition is much heavier on balafilcon A lenses than on lotrafilcon A. This can be seen in the higher average levels of all fatty acids extracted from balafilcon reported in Table 2 . Additionally, subject-to-subject variations in total lipid deposi- tion can be very significant. ${ }^{24}$ In consequence, there is considerable variation in lens-to-lens values of individual fatty acids; for given subject-lens combinations, fatty acid ratios are more relevant and reliable. One further relevant point emerges from Table 2. In all but one case, the levels of C18:1 fatty acid on continuous wear lenses are extremely low and have been expressed as "less than" values. The fact that these have been used to calculate the average C18:1-to-C18:0 ratio means in many cases the value will be significantly higher than the quoted average (ca 1:9).

One example chromatogram of each of the lens type-wear schedule combinations identified in Table 2 is shown in Figures 2 and 3.

The gas chromatograms shown in Figure 2 represent extracts of balafilcon A lenses worn on either a daily or continuous wear basis for 30 days. Chromatogram A (continuous wear balafilcon A extract) shows two predominant peaks at 12.8 and 15.0 minutes, corresponding to C16:0 and C18:0 saturated fatty acids, respectively. Little or no unsaturated fatty acid was found for the majority of continuous wear balafilcon $\mathrm{A}$ lens extracts analyzed. As shown in Table 2, Figure 2A has a 


\section{Chromatogram A: Continuous wear lotrafilcon A lens extract ( 30 days wear)}

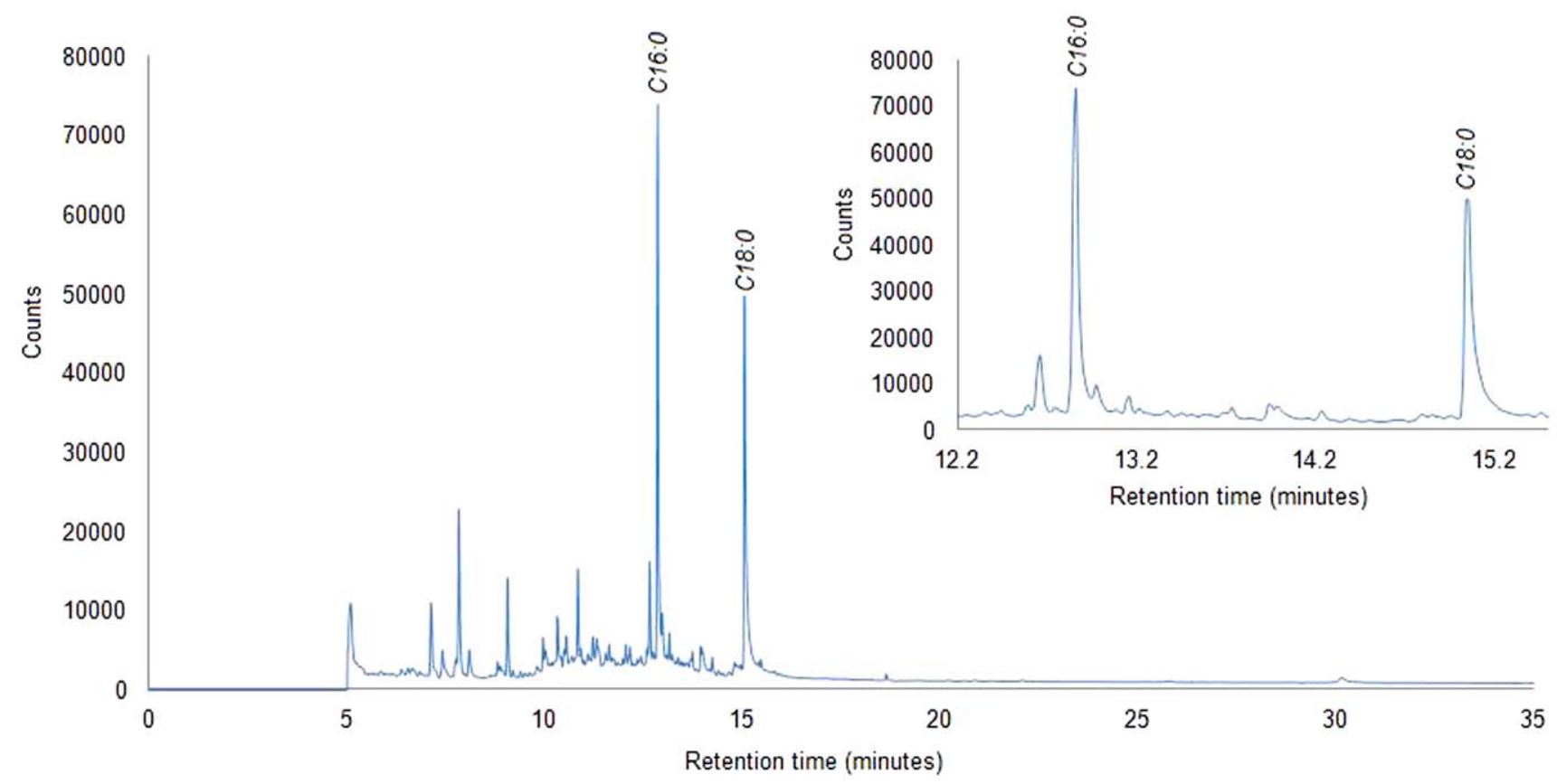

\section{Chromatogram B: Daily wear lotrafilcon A lens extract ( 30 days wear)}

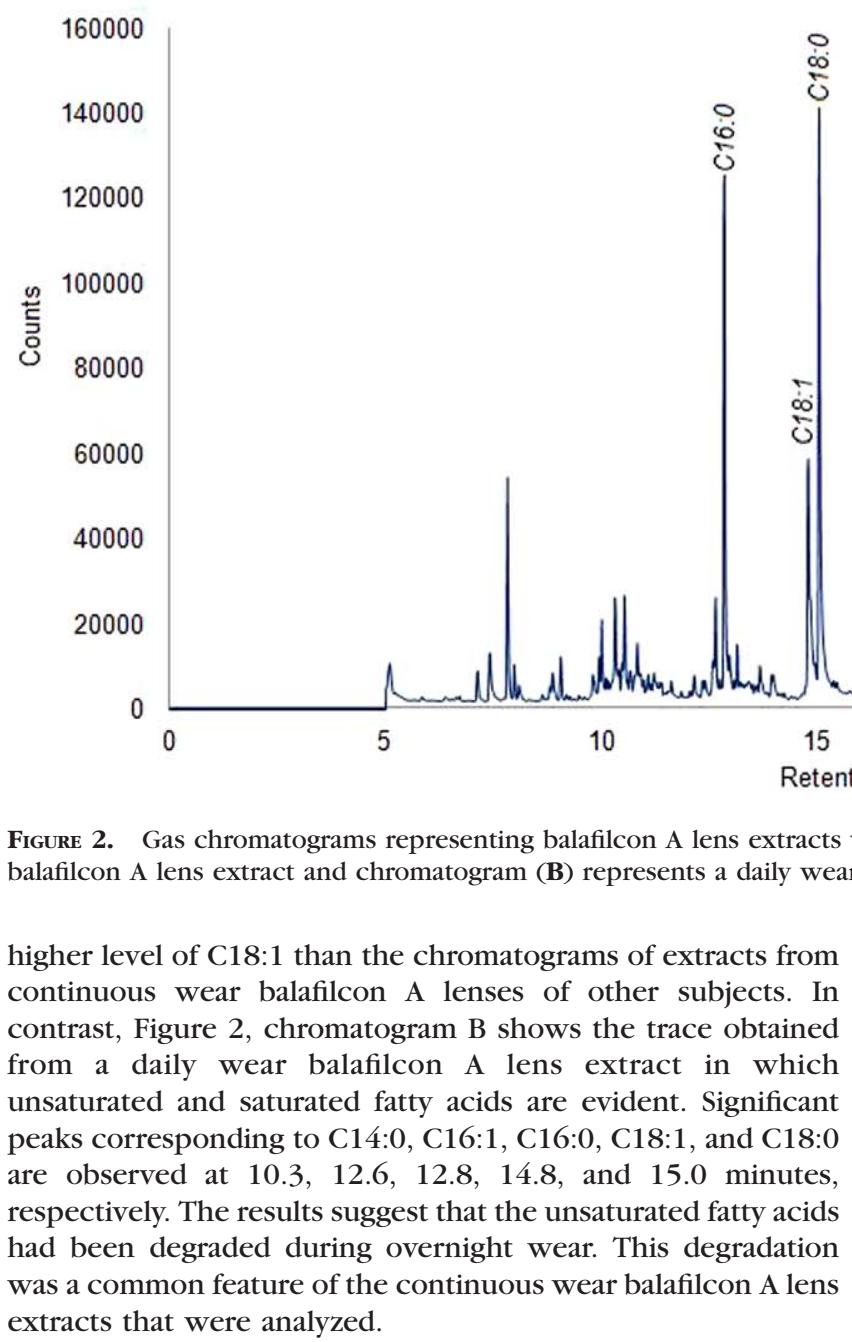

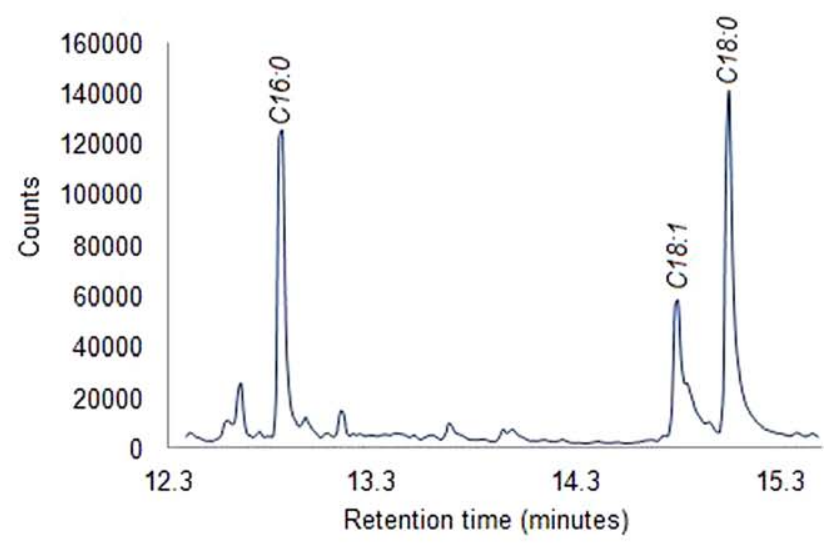




\section{Chromatogram A: Continuous wear balafilcon $A$ lens extract ( 30 days wear)}

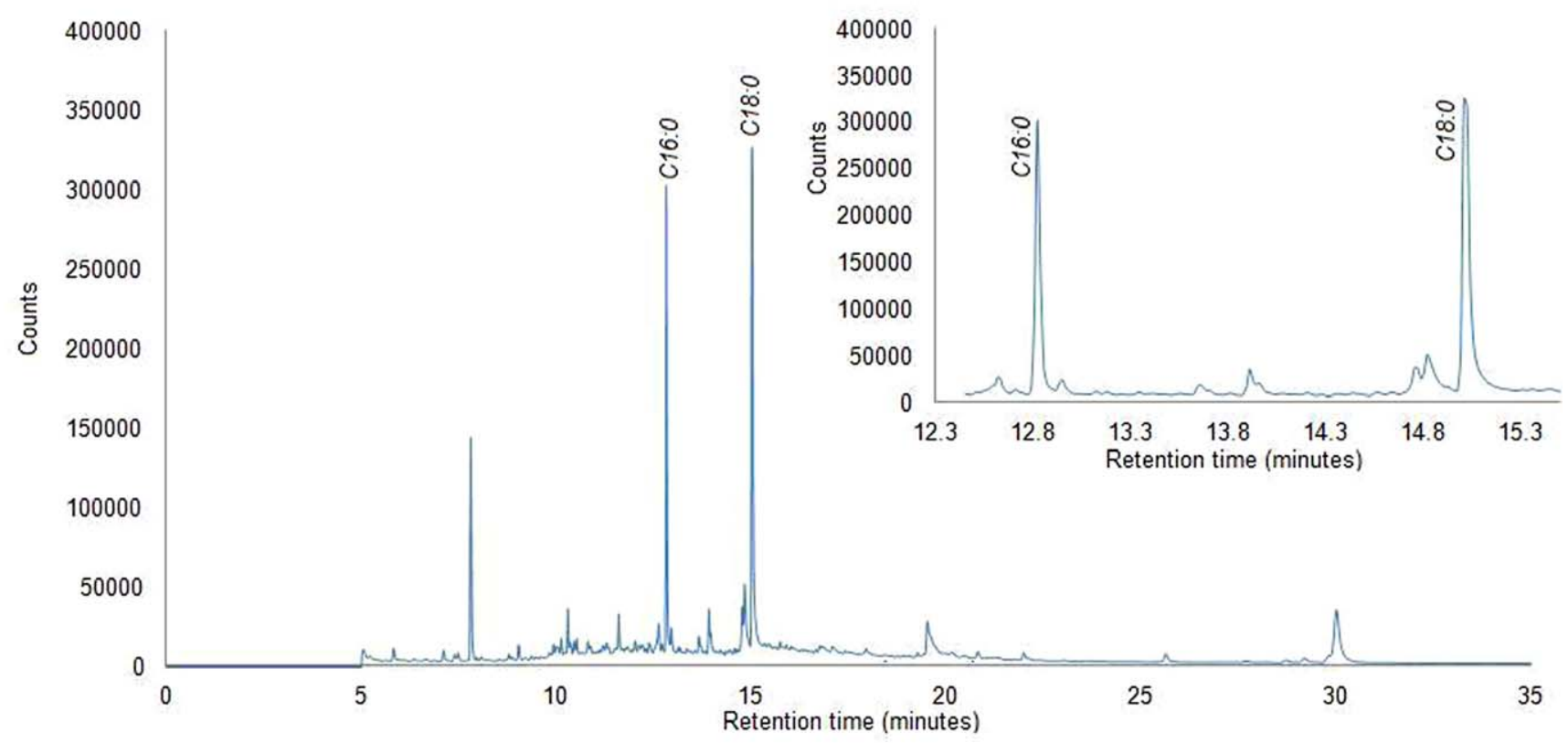

\section{Chromatogram B: Daily wear balafilcon A lens extract ( 30 days wear)}

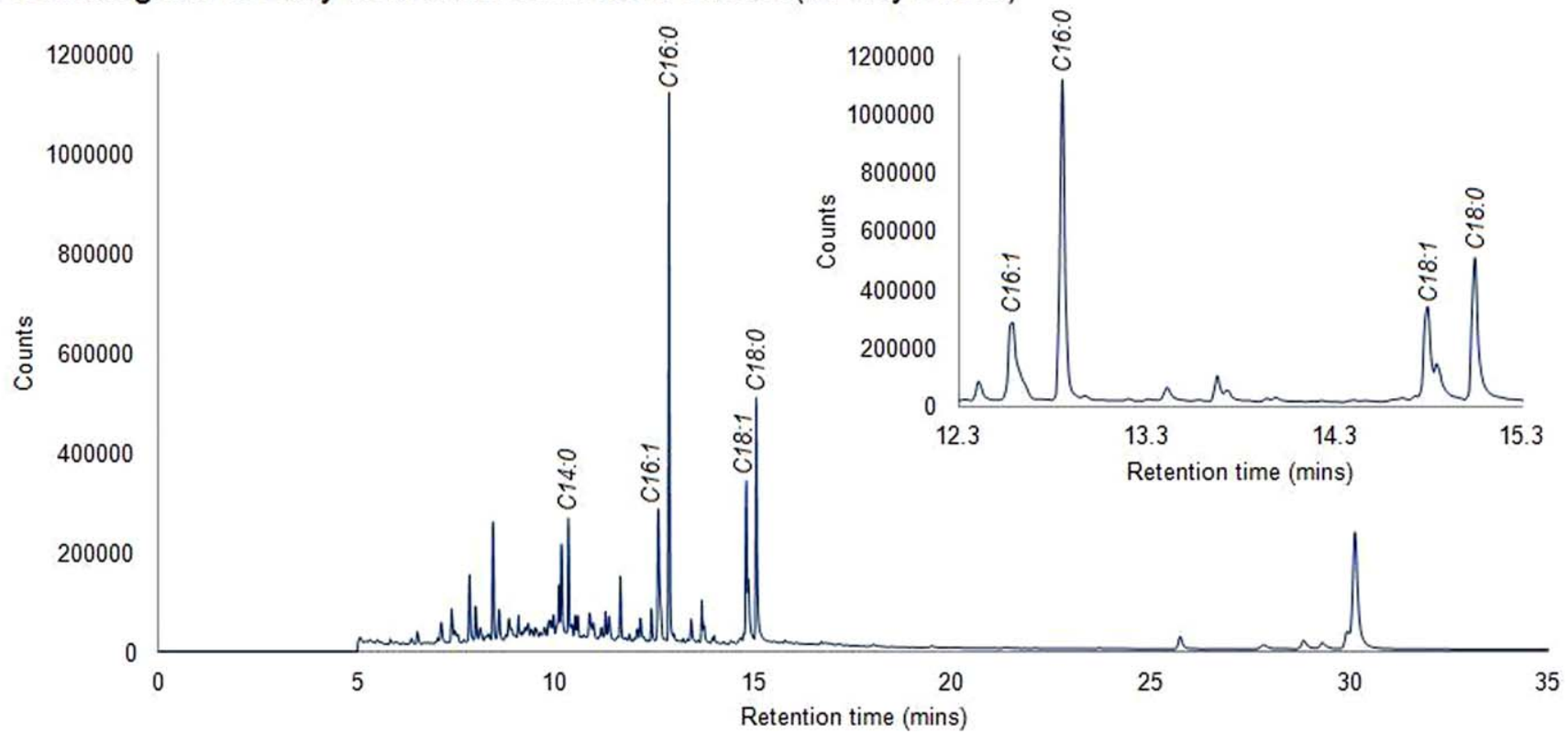

Figure 3. Gas chromatograms representing extracts of lotrafilcon A lenses worn for 30 days. Chromatogram (A) represents a continuous wear lotrafilcon A lens extract and chromatogram (B) represents a daily wear lotrafilcon A lens extract.

These results again suggested that the unsaturated fatty acids had been degraded during 30 days continuous wear.

The peak height comparisons shown in Table 3 provide a useful approach to the assessment of relative concentrations of individual fatty acids, but more detailed information can be obtained from chromatograms. Calculation of peak areas rather than peak heights and construction of calibration curves by use of external standards enables individual concentrations to be calculated.

Table 3 shows the concentrations of individual fatty acids extracted from continuous and daily wear balafilcon $\mathrm{A}$ and lotrafilcon A lenses (data collected from Figs. 2, 3). These results showed that lower levels of gross lipid were extracted from lotrafilcon A lenses compared to balafilcon A lenses. Lower levels of C16:1 (compared to C18:1) are secreted by the meibum. ${ }^{18}$ Therefore, it is logical that $\mathrm{C} 16: 1$ was not observed clearly for lotrafilcon A lens extracts $\left(<0.5 \times 10^{-4} \mathrm{mg} / \mathrm{mL}\right)$.

The data in Table 3 show a lower concentration of unsaturated compared to saturated fatty acids for these particular subjects. The levels of lipid extracted from continuous wear lenses were lower compared to daily wear lenses. While saturated lipids do not undergo oxidation in the same way as unsaturated lipids, they still are susceptible to degradation. ${ }^{35,36}$ The mechanism of degradation in the 
TABLE 3. Examples of Concentrations of Individual Fatty Acids Extracted From Single Ex Vivo Balafilcon A and Lotrafilcon A Lens Extracts Worn for 30 Days (Determined From the GC Traces Shown in Figs. 2, 3)

\begin{tabular}{lcrr}
\hline $\begin{array}{c}\text { Lens Material and } \\
\text { Wear Schedule }\end{array}$ & $\begin{array}{c}\text { Peak } \\
\text { Identity }\end{array}$ & \multicolumn{1}{c}{$\begin{array}{c}\text { Peak } \\
\text { Area }\end{array}$} & $\begin{array}{c}\text { Concentration } \\
\text { of Fatty Acid, } \\
\text { mg/mL }\end{array}$ \\
\hline Continuous wear & C16:0 & 190636 & $0.96 \times 10^{-4}$ \\
lotrafilcon A (Fig. 3, & C18:1 & 20016 & $0.1 \times 10^{-4}$ \\
chromatogram A) & C18:0 & 170514 & $0.8 \times 10^{-4}$ \\
Daily wear lotrafilcon A* & C16:0 & 349273 & $1.6 \times 10^{-4}$ \\
Fig. 3, chromatogram B) & C18:1 & 240198 & $1.2 \times 10^{-4}$ \\
& C18:0 & 436285 & $2.2 \times 10^{-4}$ \\
Continuous wear balafilcon & C16:0 & 532580 & $2.7 \times 10^{-4}$ \\
A (Fig. 2, chromatogram & $\mathrm{C} 18: 1$ & 60049 & $0.3 \times 10^{-4}$ \\
A) & $\mathrm{C} 18: 0$ & 899065 & $4.5 \times 10^{-4}$ \\
Daily wear balafilcon A $\dagger$ & $\mathrm{C} 16: 1$ & 990594 & $4.9 \times 10^{-4}$ \\
(Fig. 2, chromatogram B) & $\mathrm{C} 16: 0$ & 2119000 & $10.6 \times 10^{-4}$ \\
& $\mathrm{C} 18: 1$ & 393648 & $2.0 \times 10^{-4}$ \\
& $\mathrm{C} 18: 0$ & 1224000 & $6.2 \times 10^{-4}$ \\
\hline
\end{tabular}

* Difference in concentration of C18:1 for daily wear lotrafilcon A versus continuous wear lotrafilcon $A=1.1 \times 10-4 \mathrm{mg} / \mathrm{mL}$.

† Difference in concentration of C18:1 for daily wear balafilcon A versus continuous wear balafilcon $\mathrm{A}=1.7 \times 10-4 \mathrm{mg} / \mathrm{mL}$

overnight tear film is not fully understood as yet, but the data in Table 3 show that saturated lipids on lenses can be and are degraded during sleep. Although the oxidation of unsaturated lipids results in the production of more hydrophilic species, such as hydroperoxides, many of these are likely be solubilized in tear film components and, therefore, probably would not lead to any notable short-term alteration in the wettability of the lens.

Significant differences between daily wear and continuous wear lenses were observed, unsaturated fatty acids were substantially degraded in overnight wear lenses (and also saturated fatty acids to some extent). This pattern of unsaturated fatty acid degradation in overnight contact lens wear was observed across the range of subjects analyzed (data shown in Table 2).

Balafilcon A and lotrafilcon A lenses have very different properties, which strongly influence their lipid deposition behavior. The two lenses differ in equilibrium water content (EWC); balafilcon A lenses have an EWC of 35\% and lotrafilcon A of $24 \%$. They are silicone hydrogel lenses that have been surface treated in different ways, which leads to different surface properties, for example, balafilcon A lenses are known for higher levels of lipid deposition compared to lotrafilcon A lenses. Although balafilcon A and lotrafilcon A lenses have different characteristics, the same relative fatty acid profiles of continuous wear and daily wear lens extracts were observed. Little or no unsaturated fatty acids were extracted from lenses worn on a continuous wear schedule.

\section{Conclusions}

This research illustrates, for the first time to our knowledge, the use of GCMS for the detection, identification, and quantification of individual fatty acids extracted from single ex vivo contact lenses. It proved to be an extremely sensitive and accurate technique for the purposes of this research. Significantly, we were able to show: (1) differences in fatty acid profiles of lipids collected by various tear-sampling methods (microcapillary, ophthalmic sponge, and the lens), demonstrating that contact lenses are an effective tear-sampling method particularly for lipids; (2) a general pattern in the ratio of saturated-to-unsaturated fatty acids over a range of subjects; (3) higher levels of gross lipid on daily wear lenses compared to lenses worn on a continuous wear schedule; (4) differences between balafilcon A and lotrafilcon A lenses, primarily relating to higher levels of gross lipid on balafilcon A lenses; and (5) differences in fatty acid profiles for lenses that were worn on different wear schedules; specifically, little or no unsaturated fatty acids were associated with extracts from continuous wear lenses.

Of these findings, the most significant is the dramatic reduction in unsaturated fatty acids, after 30 days of continuous lens wear. This provides a potentially interesting means of probing the characteristics of the overnight tear film, which appeared in this study to be more effective in maintaining low levels of lipid deposition than daily wear in conjunction with the care solution used in this study (OptiFree Express).

It is difficult to predict the extent of the changes to tear lipids during sleep in the nonlens-wearing eye. It was not possible to investigate this in the context of this study, because the lens was used for the collection of lipids. The role of the contact lens wear in lipid degradation clearly is significant. Lens wear accelerates the degradation process because lipids build up on and within the lens throughout the wear schedule. A progressive build-up of unsaturated lipids on the lens leads potentially to their degradation during sleep as a result of anaerobic oxidative species in the overnight tear film. ${ }^{37}$ Although the clinical significance of these degradation processes has not been studied to our knowledge, oxidation degradation products, such as malondialdehyde, derived from more complex fatty acids, have been linked to contact lens intolerance. $^{38}$

\section{Acknowledgments}

Amandeep Panaser is grateful to the EPSRC for a research studentship.

Disclosure: A. Panaser, None; B.J. Tighe, None

\section{References}

1. Pucker AD, Nichols JJ. Analysis of meibum and tear lipids. Ocul Surf. 2012;10:230-250.

2. Iwata M, Ohno S, Kawai T, Ichijima $\mathrm{H}$, Cavanagh $\mathrm{HD}$. In vitro evaluation of lipids adsorbed on silicone hydrogel contact lenses using a new gas chromatography/mass spectrometry analytical method. Eye Contact Lens. 2008;34:272-280.

3. Butovich IA, Wojtowicz JC. Human tear film and meibum. Very long chain wax esters and (O-acyl)-omega-hydroxy fatty acids of meibum. J Lipid Res. 2009;50:2471-2485.

4. Campbell D, Griffiths G, Tighe BJ. Tear analysis and lens-tear interactions: part II. Ocular lipids-nature and fate of meibomian gland phospholipids. Cornea. 2011;30:325-332.

5. Borchman D, Foulks GN, Yappert MC, Ho DV. Temperatureinduced conformational changes in human tear lipids hydrocarbon chains. Biopolymers. 2007;87:124-133.

6. Borchman D, Foulks GN, Yappert MC, Tang D, Hoa DV. Spectroscopic evaluation of human tear lipids. Chem Phys Lipids. 2007; 147:87-102.

7. Borchman D, Yappert MC, Foulks GN. Changes in human meibum lipid with meibomian gland dysfunction using principle component analysis. Exp Eye Res. 2010;91:246-256.

8. Bron AJ, Tiffany JM. The meibomian glands and tear lipidsstructure, function and control. In: Bron AJ, Tiffany JM, eds. Lacrimal Gland, Tear Film and Dry Eye Syndromes 2. New York, NY: Springer; 1998:281-295. 
9. Bron AJ, Tiffanya JM, Gouveia SM, Yokoi N, Voon LW. Functional aspects of the tear film lipid layer. Exp Eye Res. 2004;78:347-360.

10. Butovich IA. The meibomian puzzle: combining pieces together. Prog Retin Eye Res. 2009;1-16.

11. Butovich IA. Cholesteryl esters as a depot for very long chain fatty acids in human meibum. J Lipid Res. 2009;50:501-513.

12. Butovich IA. Fatty acid composition of cholesteryl esters of human meibomian gland secretions. Steroids. 2010;75:726733.

13. Butovich IA. Lipidomics of human meibomian gland secretions: chemistry, biophysics, and physiological role of meibomian lipids. Prog Lipid Res. 2011;50:278-301.

14. Butovich IA, Millar TJ, Ham BM. Understanding and analyzing meibomian lipids-a review. Curr Eye Res. 2008;33:405-420.

15. Butovich IA, Uchiyama E, McCulley JP. Lipids of human meibum: mass-spectrometric analysis and structural elucidation. J Lipid Res. 2007;48:2220-2235.

16. Butovich IA, Uchiyama E, Pascuale MAD, McCulley JP. Liquid chromatography-mass spectrometric analysis of lipids present in human meibomian gland secretions. Am Oil Chem Soc. 2007; $42: 765-776$

17. Harvey DJ, Tiffany JM, Duerden JM, Pandher KS, Mengher LS. Identification by combined gas chromatography mass spectrometry of constituent long-chain fatty acids and alcohols from the meibomian glands of rats and a comparison with human meibomian glands. J Chromatogr. 1987;414:253-263.

18. Nicolaides N, Kaitaranta JK, Rawdah TN, Macy JI, Boswell FM III, Smith RE. Meibomian gland studies: comparison of steer and human lipids. Invest Ophthalmol Vis Sci. 1981;20:522536.

19. Butovich IA. Tear film lipids. Exp Eye Res. 2013;117:4-27.

20. Dougherty JM, McCulley JP. Analysis of the free fatty acid component of meibomian secretions in chronic blepharitis. Invest Ophthalmol Vis Sci. 1986;27:52-56.

21. Joffre C, Souchier M, Grégoire S, et al. Differences in meibomian fatty acid composition in patients with meibomian gland dysfunction and aqueous-deficient dry eye. $\mathrm{Br} J$ Ophthalmol. 2008;92:116-119.

22. Nichols KK, Ham BM, Nichols JJ, Ziegler C, Green-Church KB. Identification of fatty acids and fatty acid amides in human meibomian gland secretions. Invest Ophthalmol Vis Sci. 2007; 48:34-39.

23. Nicolaides N, Ruth EC. Unusual fatty acids in the lipids of steer and human meibomian gland excreta. Curr Eye Res. 1982;2: 93-98.
24. Panaser A, Tighe BJ. Function of lipids-their fate in contact lens wear: an interpretive review. Cont Lens Ant Eye. 2012;35: 100-111.

25. Shin H-Y, Lim S-M, Bae S-Y, Oh S-C. Thermal degradation and stability of fatty acid methyl esters in supercritical methanol. $J$ Anal App Pyrol. 2011;92:332-338.

26. Sack RA, Tan KO, Tan A. Diurnal tear cycle: evidence for a nocturnal inflammatory constitutive tear fluid. Invest $O p h$ thalmol Vis Sci. 1992;33:626-640.

27. Stapleton F, Willcox MDP, Morris CA, Sweeney DF. Tear changes in contact lens wearers following overnight eye closure. Curr Eye Res. 1997;17:183-188.

28. Tan KO, Sack RA, Holden BA, Swarbrick HA. Temporal sequence of changes in tear film composition during sleep. Curr Eye Res. 1993;12:1001-1007.

29. Tighe BJ, Jones L, Evans K, Franklin V. Patient dependant and material dependant factors in contact lens deposition processes. Adv Exp Med Biol. 1998;438:745-751.

30. Hart DE. Lipid deposits which can form on extended wear contact lenses. Int Cont Lens Clin. 1984;11:348-360.

31. Hart DE, Lane BC, Josephson JE, et al. Spoilage of hydrogel contact lenses by lipid deposits. Tear film potassium depression, fat, protein and alcohol consumption. Ophthalmology. 1987;94:1315-1321.

32. Hart DE, Tisdale RR, Sack RA. Origin and composition of lipid deposits on soft contact lenses. Ophthalmology. 1986;93:495503.

33. Pucker A, Thangavelu M, Nichols JJ. In vitro lipid deposition on hydrogel and silicone hydrogel contact lenses. Invest Ophthalmol Vis Sci. 2010;51:6334-6340.

34. Hatou S, Fukui M, Yatsui K, Mochizuki H, Akune Y, Yamada M. Biochemical analyses of lipids deposited on silicone hydrogel lenses. J Optom. 2010;3:164-168.

35. Hui SP, Sakurai T, Ohkawa F, et al. Detection and charaterization of cholesteryl ester hydroperoxides in oxidized LDL and oxidized HDL by use of an Orbitrap mass spectrometer. Anal Bioanal Chem. 2012;404:101-112.

36. Berg JM, Tymoczko JL, Stryer L. Fatty acid metabolism. In: Biochemistry. 5th ed. New York: WH Freeman; 2002:863-908.

37. Thakur A, Willcox MDP. Cytokine and lipid inflammatory mediator profile of human tears during contact lens associated inflammatory diseases. Exp Eye Res. 1998;67:9-19.

38. Glasson MJ, Stapleton F, Willcox MDP. Lipid, lipase and lipocalin differences between tolerant and intolerant contact lens wearers. Curr Eye Res. 2002;25:227-235. 